\title{
Dialectical Logic K-model: On Multidimensional Discrete Dynamical Sampling System and Further Properties of Kirchhoff Matrices
}

\author{
Yaozhi Jiang $^{1}$ \\ ${ }^{1}$ Shijiazhuang High-Tech District, Hebei, China \\ Correspondence: Yaozhi Jiang, Shijiazhuang High-Tech District, Hebei, China. E-mail: jiangyaozhi@126.com
}

Received: February 11, 2018 Accepted: March 12, 2018 Online Published: March 19, 2018

doi:10.5539/jmr.v10n3p20

URL: https://doi.org/10.5539/jmr.v10n3p20

\begin{abstract}
In order to solve the problem of multidimensional logic variable true value function remarked in the paper(Yaozhi Jiang., 2017), now author has used discrete multiple Fourier transform to deal with the problem remarked above, and obtained an theoretical formulations of discrete multidimensional Fourier transform for that multidimensional logic variable true value function is unknown or we need the frequency properties of multidimensional logic variable true value function. Another problem is about further and deeper properties of Kirchhoff matrices defined in author's paper(Yaozhi Jiang., 2017), author has established a series of matrix expression for Kirchhoff laws and some new properties of Kirchhoff matrices. These results are all compute-able and complicated.
\end{abstract}

Keywords: Multidimensional discrete dynamical sampling system, Discrete multiple Fourier transform, Multidimensional logic variable Kirchhoff matrices, Matrix expression of Kirchhoff flow function law, Matrix expression of Kirchhoff power function law

\section{Introduction}

In the dialectical logic K-model, the case about multidimensional logic variable is so important that we have to solve the sort of problem. As shown in author's papers(Yaozhi Jiang, 2017.; Yaozhi Jiang, 2018.), author has solved single-dimensional of these problems. To the case that multidimensional logic variable true value function is unknown or needs analysis properties in frequency domain of the multidimensional logic variable true value function, now author used the multidimensional discrete time and discrete space dynamical sampling system and discrete multiple Fourier transform to the sort of problem of multidimensional logic variable true value function and obtained some results in the section 2. Author also expanded the Kirchhoff graph and Kirchhoff matrix to the multidimensional logic variable true value function in the section 3 . These results are never heard of before.

\section{The Multidimensional Discrete Dynamical Sampling System for The Multidimensional Logic Variable True}

Value Function $T\left(A_{i}^{ \pm n}(x, y, z ; t)\right)$ and $T\left(A_{i}^{ \pm n}(w ; t)\right)$

The multidimensional logic variable true value function $T\left(A_{i}^{ \pm n}(x, y, z ; t)\right)$ is denoted to the true value function of dialectical logic variable $A_{i}^{ \pm n}(x, y, z ; t)$ involved 3-dimensional Euclidean space and 1-dimensional time, and true value function $T\left(A_{i}^{ \pm n}(w ; t)\right)$ is denoted a sort of logic variable $A_{i}^{ \pm n}(w ; t)$ acted in topology space such as river channel, highway, railway, etc. Author has explained the situation depended only on time $t$ (Yaozhi Jiang, 2017), now author will explain the situations in which true value function $T\left(A_{i}^{ \pm n}(x, y, z ; t)\right)$ and $T\left(A_{i}^{ \pm n}(w ; t)\right)$ is unknown to need discrete dynamical sampling system to determine their initial true value function or need multidimensional discrete dynamical sampling system for discrete multiple Fourier transform to analyse true value function properties in frequency domain.

In this paper, the true value function $T\left(A_{i}^{ \pm n}(x, y, z ; t)\right)$ of multidimensional logic variable $A_{i}^{ \pm n}(x, y, z ; t)$ and true value function $T\left(A_{i}^{ \pm n}(w, t)\right)$ of multidimensional logic variable $A_{i}^{ \pm n}(w ; t)$ are satisfied by the Dirichlet conditions, i.e. the Fourier transform of both the true value functions as above are all existent.

\subsection{Some Definitions, Symbols about Discrete Multidimensional Dynamical Sampling System and Discrete Multiple Fourier Transform}

Some definitions and symbols about discrete multidimensional dynamical sampling system and discrete multiple Fourier transform must be reviewed and recalled as below. 
2.1.1 Denote the Imaginary Symbol by

$$
j=\sqrt{-1}
$$

2.1.2 Unit Sampling Pulse

$$
\delta(x, y, z ; t)=\left\{\begin{array}{l}
1, \text { if } x=0, y=0, z=0 ; t=0 \\
0, \text { otherwise }
\end{array}\right.
$$

2.1.3 If the 4-dimensional True Value Function $T\left(A_{4}^{ \pm n}(x, y, z ; t)\right)$ Is Continuous at the Point $\left(x_{0}, y_{0}, z_{0} ; t_{0}\right)$, then the Pulse Sample Function at Point $\left(x_{0}, y_{0}, z_{0} ; t_{0}\right)$ is

$$
f^{\Uparrow}\left(x_{0}, y_{0}, z_{0} ; t_{0}\right)=T\left(A_{4}^{ \pm n}\left(x_{0}, y_{0}, z_{0} ; t_{0}\right)\right) \delta\left(x-x_{0}, y-y_{0}, z-z_{0} ; t-t_{0}\right)
$$

2.1.4 Truncation Function for 4-Dimensional True Value Function $T\left(A_{4}^{ \pm n}(x, y, z ; t)\right)$

$$
\chi(\Delta x, \Delta y, \Delta z ; \Delta t)=\left\{\begin{array}{l}
1, \text { if } t \in\left[-\frac{\Delta t}{2}, T_{t}-\frac{\Delta t}{2}\right] ; x \in\left[-\frac{\Delta x}{2}, T_{x}-\frac{\Delta x}{2}\right] ; \\
y \in\left[-\frac{\Delta y}{2}, T_{y}-\frac{\Delta y}{2}\right] ; z \in\left[-\frac{\Delta z}{2}, T_{z}-\frac{\Delta z}{2}\right] \\
0, \text { otherwise }
\end{array}\right.
$$

As above, $\Delta x, \Delta y, \Delta z, \Delta t$ all are sampling interval, $T_{x}, T_{y}, T_{z}, T_{t}$ all are truncated interval and $N_{x}=\frac{T_{x}}{\Delta x}$, $N_{y}=\frac{T_{y}}{\Delta y}, N_{z}=\frac{T_{z}}{\Delta z}, N_{t}=\frac{T_{t}}{\Delta t}$ all are sampling number. What shown above means that the sampling is an equal interval sampling and every time pulse is corresponding to $P=N_{x} N_{y} N_{z}$ space pulses, with another word, this is a lattice structure..

2.1.5 The Convolution

$$
C(x, y, z ; t)=f^{\Uparrow}(x, y, z ; t) \chi(\Delta x, \Delta y, \Delta z ; \Delta t)
$$

2.1.6 Discrete 4-dimensional Fourier transform $\Omega\left(T\left(A_{4}^{ \pm n}(x, y, z ; t)\right)\right)$ and its inverse Fourier transform $\Omega^{-1}\left(T^{*}(\alpha, \beta, \chi ; \delta)\right)$

$$
\begin{aligned}
& \Omega\left(T\left(A_{4}^{ \pm n}(x, y, z ; t)\right)\right)=T^{*}(\alpha, \beta, \chi ; \delta)= \\
& \sum_{x=0}^{N_{x}-1} \sum_{y=0}^{N_{y}-1} \sum_{z=0}^{N_{z}-1} \sum_{t=0}^{N_{t}-1} T\left(A_{4}^{ \pm n}(x, y, z ; t)\right) \exp \left(-j 2 \pi\left(\frac{x \alpha}{N_{x}}+\frac{y \beta}{N_{Y}}+\frac{z \chi}{N_{z}}+\frac{t \delta}{N_{t}}\right)\right)
\end{aligned}
$$

in the formula above, $\alpha=0,1,2, \cdots, N_{x}-1 ; \beta=0,1,2, \cdots, N_{y}-1 ; \chi=0,1,2, \cdots, N_{z}-1 ; \delta=0,1,2, \cdots, N_{t}-1$

$$
\begin{aligned}
& \Omega^{-1}\left(T^{*}(\alpha, \beta, \chi ; \delta)\right)= \\
& \frac{1}{N_{x} N_{y} N_{z} N_{t}} \sum_{\alpha=0}^{N_{x}-1} \sum_{\beta=0}^{N_{y}-1} \sum_{\chi=0}^{N_{z}-1} \sum_{\delta=0}^{N_{t}-1} T^{*}(\alpha, \beta, \chi ; \delta) \exp \left(j 2 \pi\left(\frac{x \alpha}{N_{x}}+\frac{y \beta}{N_{y}}+\frac{z \chi}{N_{z}}+\frac{t \delta}{N_{t}}\right)\right)
\end{aligned}
$$


in the formula above,

$$
x=0,1,2, \cdots, N_{x}-1 ; y=0,1,2, \cdots, N_{y}-1 ; z=0,1,2, \cdots N_{z}-1 ; t=0,1,2, \cdots, N_{t}-1
$$

Especially, when $N_{x}=N_{y}=N_{z}=N_{t}=M$, then

$$
\Omega\left(T\left(A_{4}^{ \pm n}(x, y, z ; t)\right)\right)=\sum_{x=0}^{M-1} \sum_{y=0}^{M-1} \sum_{z=0}^{M-1} \sum_{t=0}^{M-1} T\left(A_{4}^{ \pm n}(x, y, z ; t)\right) \exp \left(\frac{-j 2 \pi}{M}(x \alpha+y \beta+z \chi+t \delta)\right)
$$

in the formula above, $\alpha, \beta, \chi, \delta=0,1,2, \cdots, M-1$

$$
\Omega^{-1}\left(T^{*}(\alpha, \beta, \chi ; \delta)\right)=\frac{1}{M^{4}} \sum_{\alpha=0}^{M-1} \sum_{\beta=0}^{M-1} \sum_{\chi=0}^{M-1} \sum_{\delta=0}^{M-1} T^{*}(\alpha, \beta, \chi ; \delta) \exp \left(\frac{j 2 \pi}{M}(x \alpha+y \beta+\chi z+t \delta)\right)
$$

in the formula above, $x, y, z, t=0,1,2, \cdots, M-1$

2.1.7 Recurrences Formula of Discrete 4-Dimensional Fourier Transform $\Omega_{N}\left(T\left(A_{4}^{ \pm n}(x, y, z ; t)\right)\right)$ and Its Inverse $\Omega_{N}^{-1}\left(T\left(A_{4}^{ \pm n}(x, y, z ; t)\right)\right)$

if the the sampling number is increasing and the sampling number is $N_{x}, N_{y}, N_{z}, N_{t}$, then

$$
\begin{aligned}
& \Omega_{N}\left(T\left(A_{4}^{ \pm n}(x, y, z ; t)\right)\right)= \\
& \sum_{x=0}^{N_{x}-1} \sum_{y=0}^{N_{y}-1} \sum_{z=0}^{N_{z}-1 N_{t}-1} \sum_{t=0}^{ \pm} T\left(A_{4}^{ \pm n}(x, y, z ; t)\right) \exp \left(-j 2 \pi\left(\frac{x \alpha}{N_{x}}+\frac{y \beta}{N_{y}}+\frac{z \chi}{N_{z}}+\frac{t \delta}{N_{t}}\right)\right)
\end{aligned}
$$

in the formula above,

$$
\begin{aligned}
\alpha= & 0,1,2, \cdots, N_{x}-1 ; \beta=0,1,2, \cdots, N_{y}-1 ; \chi=0,1,2, \cdots, N_{z}-1 ; \delta=0,1,2, \cdots, N_{t}-1 \\
& \Omega_{N}^{-1}\left(T^{*}(\alpha, \beta, \chi ; \delta)\right)= \\
& \frac{1}{N_{x} N_{y} N_{z} N_{t}} \sum_{\alpha=0}^{N_{x}-1} \sum_{\beta=0}^{N_{y}-1} \sum_{\chi=0}^{N_{z}-1} \sum_{\delta=0}^{N_{t}-1} T^{*}(\alpha, \beta, \chi ; \delta) \exp \left(j 2 \pi\left(\frac{x \alpha}{N_{x}}+\frac{y \beta}{N_{y}}+\frac{z \chi}{N_{z}}+\frac{t \delta}{N_{t}}\right)\right)
\end{aligned}
$$

in the formula above,

$$
x=0,1,2, \cdots, N_{x}-1 ; y=0,1,2, \cdots, N_{y}-1 ; z=0,1,2, \cdots, N_{Z}-1 ; t=0,1,2, \cdots N_{t}-1
$$

If the sampling number is $\left(N_{x}+1\right),\left(N_{y}+1\right),\left(N_{z}+1\right)$ and $\left(N_{t}+1\right)$, then

$$
\Omega_{N+1}\left(T\left(A_{4}^{ \pm n}(x, y, z ; t)\right)\right)=\sum_{x=0}^{N_{x}} \sum_{y=0}^{N_{y}} \sum_{z=0}^{N_{z}} \sum_{t=0}^{N_{t}} T\left(A_{4}^{ \pm n}(x, y, z ; t)\right) \exp \left(-j 2 \pi\left(\frac{x \alpha}{N_{x}}+\frac{y \beta}{N_{y}}+\frac{z \chi}{N_{z}}+\frac{t \delta}{N_{t}}\right)\right) \text { in the }
$$

formula above,

$$
\alpha=0,1,2, \cdots, N_{x} ; \beta=0,1,2, \cdots, N_{y} ; \chi=0,1,2, \cdots, N_{z} ; \delta=0,1,2, \cdots, N_{t}
$$




$$
\begin{aligned}
& \Omega_{N+1}^{-1}\left(T^{*}(\alpha, \beta, \chi ; \delta)\right)=\frac{1}{\left(N_{x}+1\right)\left(N_{y}+1\right)\left(N_{z}+1\right)\left(N_{t}+1\right)} \\
& \sum_{\alpha=0}^{N_{x}} \sum_{\beta=0}^{N_{y}} \sum_{\chi=0}^{N_{z}} \sum_{\delta=0}^{N_{t}} T^{*}(\alpha, \beta, \chi ; \delta) \exp \left(j 2 \pi\left(\frac{x \alpha}{N_{x}+1}+\frac{y \beta}{N_{y}+1}+\frac{z \chi}{N_{z}+1}+\frac{t \delta}{N_{t}+1}\right)\right)
\end{aligned}
$$

in the formula above,

$$
x=0,1,2, \cdots, N_{x} ; y=0,1,2, \cdots, N_{y} ; z=0,1,2, \cdots, N_{z} ; t=0,1,2, \cdots, N_{t}
$$

Especially when the sampling number is $N_{x}=N_{y}=N_{z}=N_{t}=M$, then

$$
\Omega_{M}\left(T\left(A_{4}^{ \pm n}(x, y, z ; t)\right)\right)=\sum_{x=0}^{M-1} \sum_{y=0}^{M-1} \sum_{z=0}^{M-1} \sum_{t=0}^{M-1} T\left(A_{4}^{ \pm n}(x, y, z ; t)\right) \exp \left(\frac{-j 2 \pi}{M}(x \alpha+y \beta+z \chi+t \delta)\right)
$$

in the formula above, $\alpha, \beta, \chi, \delta=0,1,2, \cdots, M-1$

$$
\Omega_{M}^{-1}\left(T^{*}(\alpha, \beta, \chi ; \delta)\right)=\frac{1}{M^{4}} \sum_{\alpha=0}^{M-1} \sum_{\beta=0}^{M-1} \sum_{\chi=0}^{M-1} \sum_{\delta=0}^{M-1} T^{*}(\alpha, \beta, \chi ; \delta) \exp \left(\frac{j 2 \pi}{M}(x \alpha+y \beta+z \chi+t \delta)\right)
$$

in the formula above, $x, y, z, t=0,1,2, \cdots, M-1$

If the sampling number is $N_{x}=N_{y}=N_{z}=M+1$, then

$$
\Omega_{M+1}\left(T\left(A_{4}^{ \pm n}(x, y, z ; t)\right)\right)=\sum_{x=0}^{M} \sum_{y=0}^{M} \sum_{z=0}^{M} \sum_{t=0}^{M} T\left(A_{4}^{ \pm n}(x, y, z ; t)\right) \exp \left(\frac{-j 2 \pi}{M+1}(x \alpha+y \beta+z \chi+t \delta)\right)
$$

in the formula above, $\alpha, \beta, \chi, \delta=0,1,2, \cdots, M$

$$
\Omega_{M+1}^{-1}\left(T^{*}(\alpha, \beta, \chi ; \delta)\right)=\frac{1}{(M+1)^{4}} \sum_{\alpha=0}^{M} \sum_{\beta=0}^{M} \sum_{\chi=0}^{M} \sum_{\delta=0}^{M} T^{*}(\alpha, \beta, \chi ; \delta) \exp \left(\frac{j 2 \pi}{M+1}(x \alpha+y \beta+z \chi+t \delta)\right) \quad \text { in } \quad \text { the }
$$

formula above, $x, y, z, t=0,1,2, \cdots, M$

2.1.8 The Discrete 2-Dimensional Fourier Transform to True Value Function $T\left(A_{2}^{ \pm n}(w ; t)\right)$ of 2-Dimensional Logic

Variable $A_{2}^{ \pm n}(w ; \Delta t)$

Follow the section 2.1.2.,2.1.3., 2.1.4.,2.1.5., for the 2-dimensiontruevaluefunction we can established the formulas below

$$
\Omega\left(T\left(A_{2}^{ \pm n}(w ; t)\right)\right)=\sum_{w=0}^{N_{w}-1 N_{t}-1} \sum_{t=0}^{ \pm n} T\left(A_{2}^{ \pm n}(w ; t)\right) \exp \left(-j 2 \pi\left(\frac{w \phi}{N_{w}}+\frac{t \mu}{N_{t}}\right)\right)
$$

in the formula above, $\phi=0,1,2, \cdots, N_{w} ; \mu=0,1,2, \cdots, N_{t}$ 


$$
\Omega^{-1}\left(T^{*}(\phi ; \mu)\right)=\frac{1}{N_{w} N_{t}} \sum_{\phi=0}^{N_{w}-1 N_{t}-1} \sum_{\mu=0}^{*} T^{*}(\phi ; \mu) \exp \left(j 2 \pi\left(\frac{w \phi}{N_{w}}+\frac{t \mu}{N_{t}}\right)\right)
$$

in the formula above, $w=0,1,2, \cdots, N_{w} ; t=0,1,2, \cdots, N_{t}$

If the sampling number is increasing and the sampling number is $N_{w}$ and $N_{t}$, then the recurrences formula of discrete 2-dimensional Fourier transform is as below.

$$
\Omega_{N}\left(T\left(A_{2}^{ \pm n}(w ; t)\right)\right)=\sum_{w=0}^{N_{w}-1 N_{t}-1} \sum_{t=0}^{ \pm n} T\left(A_{2}^{ \pm n}(w ; t)\right) \exp \left(-j 2 \pi\left(\frac{w \phi}{N_{w}}+\frac{t \mu}{N_{t}}\right)\right)
$$

in the formula above, $\phi=0,1,2, \cdots, N_{w}-1 ; \mu=0,1,2, \cdots, N_{t}-1$

$$
\Omega^{-1}{ }_{N}\left(T^{*}(\phi ; \mu)\right)=\frac{1}{N_{w} N_{t}} \sum_{\phi=0}^{N_{w}-1 N_{t}-1} \sum_{\mu=0}^{*} T^{*}(\phi ; \mu) \exp \left(j 2 \pi\left(\frac{w \phi}{N_{w}}+\frac{t \mu}{N_{t}}\right)\right)
$$

in the formula above, $w=0,1,2, \cdots, N_{w}-1 ; t=0,1,2, \cdots, N_{t}-1$

If the sampling number is $N_{w}+1$ and $N_{t}+1$, then

$$
\Omega_{N+1}\left(T\left(A_{2}^{ \pm n}(w ; t)\right)\right)=\sum_{w=0}^{N_{w}} \sum_{t=0}^{N_{t}} T\left(A_{2}^{ \pm n}(w ; t)\right) \exp \left(-j 2 \pi\left(\frac{w \phi}{N_{w}+1}+\frac{t \mu}{N_{t}+1}\right)\right)
$$

in the formula above, $\phi=0,1,2, \cdots, N_{w} ; \mu=0,1,2, \cdots, N_{t}$

$$
\Omega^{-1}{ }_{N+1}\left(T^{*}(\phi ; \mu)\right)=\frac{1}{\left(N_{w}+1\right)\left(N_{t}+1\right)} \sum_{\phi=0}^{N_{w}-1 N_{t}-1} \sum_{\mu=0}^{*} T^{*}(\phi ; \mu) \exp \left(j 2 \pi\left(\frac{w \phi}{N_{w}+1}+\frac{t \mu}{N_{t}+1}\right)\right)
$$

in the formula above, $w=0,1,2, \cdots, N_{w} ; t=0,1,2, \cdots, N_{t}$

Especially if $N_{w}=N_{t}=M$, then

$$
\Omega_{M}\left(T\left(A_{2}^{ \pm n}(w ; t)\right)\right)=\sum_{w=0}^{M-1} \sum_{t=0}^{M-1} T\left(A_{2}^{ \pm n}(w ; t)\right) \exp \left(-\frac{j 2 \pi}{M}(w \phi+t \mu)\right)
$$

in the formula above, $\phi, \mu=0,1,2, \cdots, M-1$

$$
\Omega_{M}^{-1}\left(T^{*}(\phi ; \mu)\right)=\frac{1}{M^{2}} \sum_{\phi=0}^{M-1} \sum_{\mu=0}^{M-1} T^{*}(\phi ; \mu) \exp \left(\frac{j 2 \pi}{M}(w \phi+t \mu)\right)
$$

in the formula above, $w, t=0,1,2, \cdots, M-1$

The recurrences formula of discrete 2-dimensional Fourier transform and its inverse transform are as below

$$
\Omega_{M}\left(T\left(A_{2}^{ \pm n}(w ; t)\right)\right)=\sum_{w=0}^{M-1} \sum_{t=0}^{M-1} T\left(A_{2}^{ \pm n}(w ; t)\right) \exp \left(-\frac{j 2 \pi}{M}(w \phi+t \mu)\right)
$$

in the formula above, $\phi, \mu=0,1,2, \cdots, M-1$

$$
\Omega^{-1}{ }_{M}\left(T^{*}(\phi ; \mu)\right)=\frac{1}{M^{2}} \sum_{\phi=0}^{M-1} \sum_{\mu=0}^{M-1} T^{*}(\phi ; \mu) \exp \left(\frac{j 2 \pi}{M}(w \phi+t \mu)\right)
$$

in the formula above, $w, t=0,1,2, \cdots, M-1$ 


$$
\Omega_{M+1}\left(T\left(A_{2}^{ \pm n}(w ; t)\right)\right)=\sum_{w=0}^{M} \sum_{t=0}^{M} T\left(A_{2}^{ \pm n}(w ; t)\right) \exp \left(-\frac{j 2 \pi}{M+1}(w \phi+t \mu)\right)
$$

in the formula above, $\phi, \mu=0,1,2, \cdots, M$

$$
\Omega^{-1}{ }_{M+1}\left(T^{*}(\phi ; \mu)\right)=\frac{1}{(M+1)^{2}} \sum_{\phi=0}^{M} \sum_{\mu=0}^{M} T^{*}(\phi ; \mu) \exp \left(\frac{j 2 \pi}{M+1}(w \phi+t \mu)\right)
$$

in the formula above, $w, t=0,1,2, \cdots, M$

3. The Kirchhoff Graph and Kirchhoff Matrices of Multidimensional Logic Variable (Maden, A. D., Cevik, A. S., Cangul, I. N. et al. 2013)

In the last paper by author (Yaozhi Jiang, 2017), we have a series of definitions and concepts about the Kirchhoff matrix initially. In this section, we will explain the properties of Kirchhoff matrix carefully and deeply.

3.1 Definitions about Kirchhoff Graph (Yaozhi Jiang, 2017; Yaozhi Jiang, 2018)

Defines a graph $G_{K}=\left\{v_{i}, E_{i j}\right\}$, in which $v_{i}$ is the vertexes set and $E_{i j}$ is the edges set, the $v_{i}$ is denoted the

$i$ - th vertex and the $E_{i j}$ is denoted the edge conjunction between vertex $v_{i}$ and vertex $v_{j}$, if the edge exists.

A graph can be called as a Kirchhoff graph, if and only if:

The graph is a connected, directed, no self-loop, no multiple edge and Kirchhoff-weighted graph ;

A. In the graph every vertex $v_{i}$ is weighted a power function $v_{i}(t)$, in which $t$ is time variable; in the graph every edge $E_{i j}$ is weighted a power function $p_{i j}(t)$ produced by

$$
p_{i j}(t)=v_{i}(t)-v_{j}(t)
$$

In the formula above, the power function of head vertex minus the power function of tail vertex, otherwise the direction of edge is opposite and need make the direction of the edge redirected by another direction or sign a negative sign before the $p_{i j}(t)$;

B. In the graph, there is a pair of vertex, one vertex is called as source vertex $v_{+1}$, its power function is constant +1 , and another vertex is called as sink vertex $v_{-1}$, its power function is constant -1 , and the power function of the edge $E_{+1,-1}$ is a constant 2, the flow function of edge $E_{+1,-1}$ is a constant $I$;

C. In the graph, for $k$-dimensional logic variable there is a complete sub-graph, its vertex number is $2 k$, of cause its edge number is $k(2 k-1)$. This complete sub-graph can be called as primary sub-graph;

D. In the graph, for $n$-contradiction factors there are $k(2 k-1)$ secondary sub-graph (See Yaozhi Jiang 2018);

E. The source vertex $v_{+1}$ is connected to every positive vertex, and the sink vertex $v_{-1}$ is connected to every negative vertex;

F. To the every cycle $H_{m}$ in the graph, the formula as below must be satisfied:

$$
\sum_{\text {if edg } E_{i j} \in H_{m}} p_{i j}(t)=0
$$

In which $H_{m}$ is denoted the $m$ - th cycle in the graph;

G. In the graph every edge $E_{i j}$, if the edge $E_{i j}$ exists, is weighted a flow function $f_{i j}(t)$, to the total edges conjugated with vertex $v_{i}$, the formula as below is satisfied:

$$
\sum_{\text {if } f_{i j}(t) \in v_{i}} f_{i j}(t)=0
$$

In which incoming edges is positive and outgoing edges is negative;

I. The formula below is defined as a contradiction function $C_{i j}(t)$ on the edge $E_{i j}$ : 


$$
C_{i j}(t)=\frac{v_{i}(t)-v_{j}(t)}{f_{i j}(t)}=\frac{p_{i j}(t)}{f_{i j}(t)}
$$

J.Define the energy produced on the edge $E_{i j}$ or the work done on the edge $E_{i j}$ as below:

$$
w_{i j}=\int_{t_{0}}^{t_{1}} p_{i j}(t) f_{i j}(t) d t
$$

If the $w_{i j}>0$, then the definite integral above is defined as that an energy source produced on edge $E_{i j}$; if the $w_{i j}<0$, then the definite integral above is defined as that work done on the edge $E_{i j}$. Thus we have the energy conversation law as below:

$$
\sum_{\text {for-all-edges }} w_{i j}=\sum_{\text {for-all-edges } t_{0}} \int_{i j}^{t_{0}} p_{i j}(t) f_{i j}(t) d t=0
$$

i.e. the algebraic sum of energy produced on every edge and work done on every edge is zero in the graph.

Now we have a series of definitions to a sort of graph, the sort of graph can be called as Kirchhoff graph. Because of the graph is satisfied by Kirchhoff laws.

\subsection{Kirchhoff Matrix}

From definition of Kirchhoff graph above, we can obtain the Kirchhoff matrices via special adjacent matrix for Kirchhoff graph.

A. The first row of Kirchhoff power function matrix is the power function sequence below:

$$
\begin{aligned}
& +1,-1, p_{1,1}^{+}(t), p_{1,1}^{-}(t), p_{1,2}^{+}(t), p_{1,2}^{-}(t), \cdots, p_{1, n}^{+}(t), p_{1, n}^{-}(t), p_{2,1}^{+}(t), p_{2,1}^{-}(t), p_{2,2}^{+}(t), p_{2,2}^{-}(t), \cdots, \\
& p_{2, n}^{+}(t), p_{2, n}^{-}(t), \cdots, p_{p, 1}^{+}(t), p_{p, 1}^{-}(t), p_{p, 2}^{+}(t), p_{p, 2}^{-}(t), \cdots, p_{p, n}^{+}(t), p_{p, n}^{-}(t)
\end{aligned}
$$

to vertex sequence below:

$$
\begin{aligned}
& v_{+1}, v_{-1}, v_{1,1}^{+}(t), v_{1,1}^{-}(t), v_{1,2}^{+}(t), v_{1,2}^{-}(t), \cdots, v_{1, n}^{+}(t), v_{1, n}^{-}(t), v_{2,1}^{+}(t), v_{2,1}^{-}(t), v_{2,2}^{+}(t), v_{2,2}^{-}(t), \cdots, \\
& v_{2, n}^{+}(t), v_{2, n}^{-}(t), \cdots, v_{p, 1}^{+}(t), v_{p, 1}^{-}(t), v_{p, 2}^{+}(t), v_{p, 2}^{-}(t), \cdots, v_{p, n}^{+}(t), v_{p, n}^{-}(t)
\end{aligned}
$$

its first column is the transposed sequence of first row;

B. Its other elements of power function matrix is the power function $p_{i j}(t)$ of corresponding edge $E_{i j}$ (if the edge $E_{i j}$ exists);

The first row of Kirchhoff flow function matrix is the vertex sequence :

$$
\begin{aligned}
& v_{+1}, v_{-1}, v_{1,1}^{+}(t), v_{1,1}^{-}(t), v_{1,2}^{+}(t), v_{1,2}^{-}(t), \cdots, v_{1, n}^{+}(t), v_{1, n}^{-}(t), v_{2,1}^{+}(t), v_{2,1}^{-}(t), v_{2,2}^{+}(t), v_{2,2}^{-}(t), \cdots, \\
& v_{2, n}^{+}(t), v_{2, n}^{-}(t), \cdots, v_{p, 1}^{+}(t), v_{p, 1}^{-}(t), v_{p, 2}^{+}(t), v_{p, 2}^{-}(t), \cdots, v_{p, n}^{+}(t), v_{p, n}^{-}(t)
\end{aligned}
$$

its first column is the transposed sequence of first row, and other elements of flow function matrix is the flow function $f_{i j}(t)$ on corresponding edge $E_{i j}$ (if the edge $E_{i j}$ exists);

C. The contradiction function matrix is depended on the definition in A., its first row and first column are as same as the definition in A., its other elements are contradiction function

$$
C_{i j}(t)=\frac{p_{i j}(t)}{f_{i j}(t)}
$$

on corresponding edge $E_{i j}$ (if edge $E_{i j}$ exists);

D. The first row and first column of Kirchhoff power capacity function matrix are as same as the definition in A., its 
other elements are that power function $p_{i j}(t)$ times flow function $f_{i j}(t)$, i.e.

$$
p_{i j}^{c}(t)=p_{i j}(t) f_{i j}(t)
$$

on corresponding edge $E_{i j}$ (if the edge $E_{i j}$ exists);

E. The first row and first column of Kirchhoff work and energy infinite integral matrix are as same as the definition in A., its other elements are that definite integral

$$
w_{i j}=\int_{t_{0}}^{t_{1}} p_{i j}(t) f_{i j}(t) d t
$$

on corresponding edge $E_{i j}$ (if edge $E_{i j}$ exists);

So we have the further properties about Kirchhoff matrix below.

3.2.1 Power Function Matrix $M_{K p}$

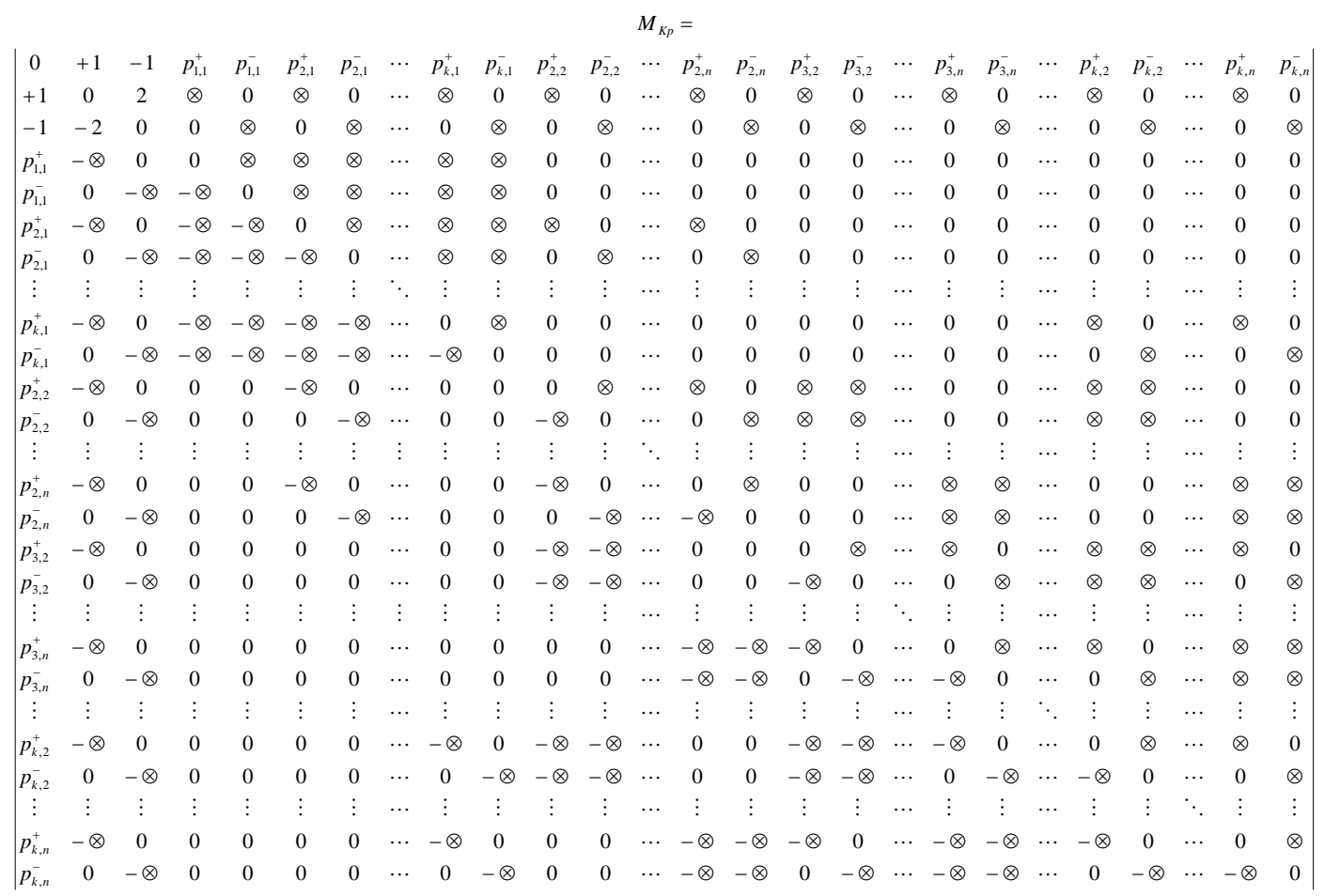

In the Kirchhoff power function matrix above, the matrix element is

$$
\otimes_{i j}=p_{i j}(t)
$$

on corresponding edges $E_{i j}$ (if the $E_{i j}$ exists). The time $t$ in vertex sequence symbols of the first row and the first column is deleted to save page space. 


\subsubsection{Flow Function Matrix $M_{K f}$}

\begin{tabular}{|c|c|c|c|c|c|c|c|c|c|c|c|c|c|c|c|c|c|c|c|c|c|c|c|c|c|}
\hline \multicolumn{26}{|c|}{$M_{K f}=$} \\
\hline 0 & $v_{+1}$ & $v_{-1}$ & $v_{1,1}^{+}$ & $v_{1,1}^{-}$ & $v_{2,1}^{+}$ & $v_{2,1}^{-}$ & $\ldots$ & $v_{k, 1}^{+}$ & $v_{k, 1}^{-}$ & $v_{2,2}^{+}$ & $v_{2,2}^{-}$ & $\ldots$ & $v_{2, n}^{+}$ & $v_{2, n}^{-}$ & $v_{3,2}^{+}$ & $v_{3,2}^{-}$ & $\ldots$ & $v_{3, n}^{+}$ & $v_{3, n}^{-}$ & $\ldots$ & $v_{k, 2}^{+}$ & $v_{k, 2}^{-}$ & $\cdots$ & $v_{k, n}^{+}$ & $v_{k, n}^{-}$ \\
\hline$v_{+1}$ & 0 & $I$ & $\otimes$ & 0 & $\otimes$ & 0 & $\ldots$ & $\otimes$ & 0 & $\otimes$ & 0 & $\ldots$ & $\otimes$ & 0 & $\otimes$ & 0 & $\ldots$ & $\otimes$ & 0 & $\ldots$ & $\otimes$ & 0 & $\ldots$ & $\otimes$ & 0 \\
\hline$v_{-1}$ & $-I$ & 0 & 0 & $\otimes$ & 0 & $\otimes$ & $\ldots$ & 0 & $\otimes$ & 0 & $\otimes$ & $\ldots$ & 0 & $\otimes$ & 0 & $\otimes$ & $\ldots$ & 0 & $\otimes$ & $\ldots$ & 0 & $\otimes$ & $\ldots$ & 0 & $\otimes$ \\
\hline$v_{1,1}^{+}$ & $-\otimes$ & 0 & 0 & $\otimes$ & $\otimes$ & $\otimes$ & $\ldots$ & $\otimes$ & $\otimes$ & 0 & 0 & $\ldots$ & 0 & 0 & 0 & 0 & $\ldots$ & 0 & 0 & $\ldots$ & 0 & 0 & $\ldots$ & 0 & 0 \\
\hline$v_{1,1}^{-}$ & 0 & $-\otimes$ & $-\otimes$ & 0 & $\otimes$ & $\otimes$ & $\ldots$ & $\otimes$ & $\otimes$ & 0 & 0 & $\ldots$ & 0 & 0 & 0 & 0 & $\ldots$ & 0 & 0 & $\ldots$ & 0 & 0 & $\ldots$ & 0 & 0 \\
\hline$v_{2,1}^{+}$ & $-\otimes$ & 0 & $-\otimes$ & $-\otimes$ & 0 & $\otimes$ & $\cdots$ & $\otimes$ & $\otimes$ & $\otimes$ & 0 & $\ldots$ & $\otimes$ & 0 & 0 & 0 & $\ldots$ & 0 & 0 & $\ldots$ & 0 & 0 & $\ldots$ & 0 & 0 \\
\hline$v_{2,1}^{-}$ & 0 & $-\otimes$ & $-\otimes$ & $-\otimes$ & $-\otimes$ & 0 & $\ldots$ & $\otimes$ & $\otimes$ & 0 & $\otimes$ & $\ldots$ & 0 & $\otimes$ & 0 & 0 & $\ldots$ & 0 & 0 & $\ldots$ & 0 & 0 & $\ldots$ & 0 & 0 \\
\hline$\vdots$ & $\vdots$ & $\vdots$ & $\vdots$ & $\vdots$ & $\vdots$ & $\vdots$ & $\ddots$ & $\vdots$ & $\vdots$ & $\vdots$ & $\vdots$ & $\ldots$ & $\vdots$ & $\vdots$ & $\vdots$ & $\vdots$ & $\ldots$ & $\vdots$ & $\vdots$ & $\ldots$ & $\vdots$ & $\vdots$ & $\ldots$ & $\vdots$ & $\vdots$ \\
\hline$v_{k, 1}^{+}$ & $-\otimes$ & 0 & $-\otimes$ & $-\otimes$ & $-\otimes$ & $-\otimes$ & $\ldots$ & 0 & $\otimes$ & 0 & 0 & $\ldots$ & 0 & 0 & 0 & 0 & $\ldots$ & 0 & 0 & $\ldots$ & $\otimes$ & 0 & $\ldots$ & $\otimes$ & 0 \\
\hline$v_{k, 1}^{-}$ & 0 & $-\otimes$ & $-\otimes$ & $-\otimes$ & $-\otimes$ & $-\otimes$ & $\ldots$ & $-\otimes$ & 0 & 0 & 0 & $\ldots$ & 0 & 0 & 0 & 0 & $\ldots$ & 0 & 0 & $\ldots$ & 0 & $\otimes$ & $\ldots$ & 0 & $\otimes$ \\
\hline$v_{2,2}^{+}$ & $-\otimes$ & 0 & 0 & 0 & $-\otimes$ & 0 & $\ldots$ & 0 & 0 & 0 & $\otimes$ & $\ldots$ & $\otimes$ & 0 & $\otimes$ & $\otimes$ & $\ldots$ & 0 & 0 & $\ldots$ & $\otimes$ & $\otimes$ & $\cdots$ & 0 & 0 \\
\hline$v_{2,2}^{-}$ & 0 & $-\otimes$ & 0 & 0 & 0 & $-\otimes$ & $\ldots$ & 0 & 0 & $-\otimes$ & 0 & $\ldots$ & 0 & $\otimes$ & $\otimes$ & $\otimes$ & $\ldots$ & 0 & 0 & $\ldots$ & $\otimes$ & $\otimes$ & $\ldots$ & 0 & 0 \\
\hline$\vdots$ & $\vdots$ & $\vdots$ & $\vdots$ & $\vdots$ & $\vdots$ & $\vdots$ & $\vdots$ & $\vdots$ & $\vdots$ & $\vdots$ & $\vdots$ & $\ddots$ & $\vdots$ & $\vdots$ & $\vdots$ & $\vdots$ & $\ldots$ & $\vdots$ & $\vdots$ & $\ldots$ & $\vdots$ & $\vdots$ & $\ldots$ & $\vdots$ & $\vdots$ \\
\hline$v_{2, n}^{+}$ & $-\otimes$ & 0 & 0 & 0 & $-\otimes$ & 0 & $\ldots$ & 0 & 0 & $-\otimes$ & 0 & $\ldots$ & 0 & $\otimes$ & 0 & 0 & $\ldots$ & $\otimes$ & $\otimes$ & $\ldots$ & 0 & 0 & $\ldots$ & $\otimes$ & $\otimes$ \\
\hline$v_{2, n}^{-}$ & 0 & $-\otimes$ & 0 & 0 & 0 & $-\otimes$ & $\ldots$ & 0 & 0 & 0 & $-\otimes$ & $\ldots$ & $-\otimes$ & 0 & 0 & 0 & $\ldots$ & $\otimes$ & $\otimes$ & $\ldots$ & 0 & 0 & $\ldots$ & $\otimes$ & $\otimes$ \\
\hline$v_{3,2}^{+}$ & $-\otimes$ & 0 & 0 & 0 & 0 & 0 & $\ldots$ & 0 & 0 & $-\otimes$ & $-\otimes$ & $\ldots$ & 0 & 0 & 0 & $\otimes$ & $\ldots$ & $\otimes$ & 0 & $\ldots$ & $\otimes$ & $\otimes$ & $\ldots$ & $\otimes$ & 0 \\
\hline$v_{3,2}^{-}$ & 0 & $-\otimes$ & 0 & 0 & 0 & 0 & $\ldots$ & 0 & 0 & $-\otimes$ & $-\otimes$ & $\ldots$ & 0 & 0 & $-\otimes$ & 0 & $\ldots$ & 0 & $\otimes$ & $\ldots$ & $\otimes$ & $\otimes$ & $\ldots$ & 0 & $\otimes$ \\
\hline$\vdots$ & $\vdots$ & $\vdots$ & $\vdots$ & $\vdots$ & $\vdots$ & $\vdots$ & $\vdots$ & $\vdots$ & $\vdots$ & $\vdots$ & $\vdots$ & $\ldots$ & $\vdots$ & $\vdots$ & $\vdots$ & $\vdots$ & $\ddots$ & $\vdots$ & $\vdots$ & $\ldots$ & $\vdots$ & $\vdots$ & $\ldots$ & $\vdots$ & $\vdots$ \\
\hline$v_{3, n}^{+}$ & $-\otimes$ & 0 & 0 & 0 & 0 & 0 & $\ldots$ & 0 & 0 & 0 & 0 & $\ldots$ & $-\otimes$ & $-\otimes$ & $-\otimes$ & 0 & $\ldots$ & 0 & $\otimes$ & $\ldots$ & 0 & 0 & $\ldots$ & $\otimes$ & $\otimes$ \\
\hline$v_{3, n}^{-}$ & 0 & $-\otimes$ & 0 & 0 & 0 & 0 & $\ldots$ & 0 & 0 & 0 & 0 & $\ldots$ & $-\otimes$ & $-\otimes$ & 0 & $-\otimes$ & $\ldots$ & $-\otimes$ & 0 & $\ldots$ & 0 & 0 & $\ldots$ & $\otimes$ & $\otimes$ \\
\hline$\vdots$ & $\vdots$ & $\vdots$ & $\vdots$ & $\vdots$ & $\vdots$ & $\vdots$ & $\ldots$ & $\vdots$ & $\vdots$ & $\vdots$ & $\vdots$ & $\ldots$ & $\vdots$ & $\vdots$ & $\vdots$ & $\vdots$ & $\ldots$ & $\vdots$ & $\vdots$ & $\ddots$ & $\vdots$ & $\vdots$ & $\ldots$ & $\vdots$ & $\vdots$ \\
\hline$v_{k, 2}^{+}$ & $-\otimes$ & 0 & 0 & 0 & 0 & 0 & $\ldots$ & $-\otimes$ & 0 & $-\otimes$ & $-\otimes$ & $\ldots$ & 0 & 0 & $-\otimes$ & $-\otimes$ & $\ldots$ & $\otimes$ & 0 & $\ldots$ & 0 & $\otimes$ & $\ldots$ & $\otimes$ & 0 \\
\hline$v_{k, 2}^{-}$ & 0 & $-\otimes$ & 0 & 0 & 0 & 0 & $\ldots$ & 0 & $-\otimes$ & $-\otimes$ & $-\otimes$ & $\ldots$ & 0 & 0 & $-\otimes$ & $-\otimes$ & $\ldots$ & 0 & $\otimes$ & $\cdots$ & $-\otimes$ & 0 & $\ldots$ & 0 & $\otimes$ \\
\hline$\vdots$ & $\vdots$ & $\vdots$ & $\vdots$ & $\vdots$ & $\vdots$ & $\vdots$ & $\ldots$ & $\vdots$ & $\vdots$ & $\vdots$ & $\vdots$ & $\ldots$ & $\vdots$ & $\vdots$ & $\vdots$ & $\vdots$ & $\ldots$ & $\vdots$ & $\vdots$ & $\ldots$ & $\vdots$ & $\vdots$ & $\ddots$ & $\vdots$ & $\vdots$ \\
\hline$v_{k, n}^{+}$ & $-\otimes$ & 0 & 0 & 0 & 0 & 0 & $\ldots$ & $-\otimes$ & 0 & 0 & 0 & $\ldots$ & $-\otimes$ & $-\otimes$ & $-\otimes$ & 0 & $\ldots$ & $-\otimes$ & $-\otimes$ & $\ldots$ & $-\otimes$ & 0 & $\ldots$ & 0 & $\otimes$ \\
\hline$v_{k, n}^{-}$ & 0 & $-\otimes$ & 0 & 0 & 0 & 0 & $\ldots$ & 0 & $-\otimes$ & 0 & 0 & $\ldots$ & $-\otimes$ & $-\otimes$ & 0 & $-\otimes$ & $\ldots$ & $-\otimes$ & $-\otimes$ & $\ldots$ & 0 & $-\otimes$ & $\ldots$ & $-\otimes$ & 0 \\
\hline
\end{tabular}

In the Kirchhoff flow function matrix above, the element of the matrix is every flow function

$$
\otimes_{i j}=f_{i j}(t)
$$

on the corresponding edge $E_{i j}$ (if the edge $E_{i j}$ exists). The time $t$ in vertex sequence symbols of the first row and the first column is deleted to save page space. 
3.2.3 Contradiction Function Matrix $M_{K c}=M_{K p} / M_{K f}$

\begin{tabular}{|c|c|c|c|c|c|c|c|c|c|c|c|c|c|c|c|c|c|c|c|c|c|c|c|c|c|}
\hline \multicolumn{26}{|c|}{$M_{K c}=$} \\
\hline 0 & $v_{+1}$ & $v_{-1}$ & $v_{1,1}^{+}$ & $v_{1,1}^{-}$ & $v_{2,1}^{+}$ & $v_{2,1}^{-}$ & $\cdots$ & $v_{k, 1}^{+}$ & $v_{k, 1}^{-}$ & $v_{2,2}^{+}$ & $v_{2,2}^{-}$ & $\cdots$ & $v_{2, n}^{+}$ & $v_{2, n}^{-}$ & $v_{3,2}^{+}$ & $v_{3,2}^{-}$ & $\cdots$ & $v_{3, n}^{+}$ & $v_{3, n}^{-}$ & $\cdots$ & $v_{k, 2}^{+}$ & $v_{k, 2}^{-}$ & $\cdots$ & $v_{k, n}^{+}$ & $v_{k, n}^{-}$ \\
\hline$v_{+1}$ & 0 & $2 / I$ & $\otimes$ & 0 & $\otimes$ & 0 & $\cdots$ & $\otimes$ & 0 & $\otimes$ & 0 & $\cdots$ & $\otimes$ & 0 & $\otimes$ & 0 & $\cdots$ & $\otimes$ & 0 & $\cdots$ & $\otimes$ & 0 & $\cdots$ & $\otimes$ & 0 \\
\hline$v_{-1}$ & $-2 / I$ & 0 & 0 & $\otimes$ & 0 & $\otimes$ & $\ldots$ & 0 & $\otimes$ & 0 & $\otimes$ & $\cdots$ & 0 & $\otimes$ & 0 & $\otimes$ & $\cdots$ & 0 & $\otimes$ & $\ldots$ & 0 & $\otimes$ & $\ldots$ & 0 & $\otimes$ \\
\hline$v_{1,1}^{+}$ & $-\otimes$ & 0 & 0 & $\otimes$ & $\otimes$ & $\otimes$ & $\ldots$ & $\otimes$ & $\otimes$ & 0 & 0 & $\ldots$ & 0 & 0 & 0 & 0 & $\ldots$ & 0 & 0 & $\ldots$ & 0 & 0 & $\ldots$ & 0 & 0 \\
\hline$v_{1,1}^{-}$ & 0 & $-\otimes$ & $-\otimes$ & 0 & $\otimes$ & $\otimes$ & $\ldots$ & $\otimes$ & $\otimes$ & 0 & 0 & $\ldots$ & 0 & 0 & 0 & 0 & $\ldots$ & 0 & 0 & $\ldots$ & 0 & 0 & $\ldots$ & 0 & 0 \\
\hline$v_{2,1}^{+}$ & $-\otimes$ & 0 & $-\otimes$ & $-\otimes$ & 0 & $\otimes$ & $\ldots$ & $\otimes$ & $\otimes$ & $\otimes$ & 0 & $\ldots$ & $\otimes$ & 0 & 0 & 0 & $\ldots$ & 0 & 0 & $\ldots$ & 0 & 0 & $\ldots$ & 0 & 0 \\
\hline$v_{2,1}^{-}$ & 0 & $-\otimes$ & $-\otimes$ & $-\otimes$ & $-\otimes$ & 0 & $\ldots$ & $\otimes$ & $\otimes$ & 0 & $\otimes$ & $\ldots$ & 0 & $\otimes$ & 0 & 0 & $\ldots$ & 0 & 0 & $\ldots$ & 0 & 0 & $\ldots$ & 0 & 0 \\
\hline$\vdots$ & $\vdots$ & $\vdots$ & $\vdots$ & $\vdots$ & $\vdots$ & $\vdots$ & $\ddots$ & $\vdots$ & $\vdots$ & $\vdots$ & $\vdots$ & $\ldots$ & $\vdots$ & $\vdots$ & $\vdots$ & $\vdots$ & $\ldots$ & $\vdots$ & $\vdots$ & $\ldots$ & $\vdots$ & $\vdots$ & $\ldots$ & $\vdots$ & $\vdots$ \\
\hline$v_{k, 1}^{+}$ & $-\otimes$ & 0 & $-\otimes$ & $-\otimes$ & $-\otimes$ & $-\otimes$ & $\ldots$ & 0 & $\otimes$ & 0 & 0 & $\ldots$ & 0 & 0 & 0 & 0 & $\ldots$ & 0 & 0 & $\ldots$ & $\otimes$ & 0 & $\ldots$ & $\otimes$ & 0 \\
\hline$v_{k, 1}^{-}$ & 0 & $-\otimes$ & $-\otimes$ & $-\otimes$ & $-\otimes$ & $-\otimes$ & $\ldots$ & $-\otimes$ & 0 & 0 & 0 & $\ldots$ & 0 & 0 & 0 & 0 & $\ldots$ & 0 & 0 & $\ldots$ & 0 & $\otimes$ & $\ldots$ & 0 & $\otimes$ \\
\hline$v_{2,2}^{+}$ & $-\otimes$ & 0 & 0 & 0 & $-\otimes$ & 0 & $\ldots$ & 0 & 0 & 0 & $\otimes$ & $\ldots$ & $\otimes$ & 0 & $\otimes$ & $\otimes$ & $\ldots$ & 0 & 0 & $\ldots$ & $\otimes$ & $\otimes$ & $\ldots$ & 0 & 0 \\
\hline$v_{2,2}^{-}$ & 0 & $-\otimes$ & 0 & 0 & 0 & $-\otimes$ & $\ldots$ & 0 & 0 & $-\otimes$ & 0 & $\ldots$ & 0 & $\otimes$ & $\otimes$ & $\otimes$ & $\ldots$ & 0 & 0 & $\ldots$ & $\otimes$ & $\otimes$ & $\ldots$ & 0 & 0 \\
\hline & $\vdots$ & $\vdots$ & $\vdots$ & $\vdots$ & $\vdots$ & $\vdots$ & $\vdots$ & $\vdots$ & $\vdots$ & $\vdots$ & $\vdots$ & $\ddots$ & $\vdots$ & $\vdots$ & $\vdots$ & $\vdots$ & $\ldots$ & $\vdots$ & $\vdots$ & $\ldots$ & $\vdots$ & $\vdots$ & $\ldots$ & $\vdots$ & \\
\hline$v_{2, n}^{+}$ & $-\otimes$ & 0 & 0 & 0 & $-\otimes$ & 0 & $\ldots$ & 0 & 0 & $-\otimes$ & 0 & $\ldots$ & 0 & $\otimes$ & 0 & 0 & $\ldots$ & $\otimes$ & $\otimes$ & $\ldots$ & 0 & 0 & $\ldots$ & $\otimes$ & $\otimes$ \\
\hline$v_{2, n}^{-}$ & 0 & $-\otimes$ & 0 & 0 & 0 & $-\otimes$ & $\ldots$ & 0 & 0 & 0 & $-\otimes$ & $\ldots$ & $-\otimes$ & 0 & 0 & 0 & $\ldots$ & $\otimes$ & $\otimes$ & $\ldots$ & 0 & 0 & $\ldots$ & $\otimes$ & $\otimes$ \\
\hline$v_{3,3}^{+}$ & $-\otimes$ & 0 & 0 & 0 & 0 & 0 & $\ldots$ & 0 & 0 & $-\otimes$ & $-\otimes$ & $\ldots$ & 0 & 0 & 0 & $\otimes$ & $\ldots$ & $\otimes$ & 0 & $\ldots$ & $\otimes$ & $\otimes$ & $\ldots$ & $\otimes$ & 0 \\
\hline$v_{32}^{-}$ & 0 & $-\otimes$ & 0 & 0 & 0 & 0 & $\ldots$ & 0 & 0 & $-\otimes$ & $-\otimes$ & $\ldots$ & 0 & 0 & $-\otimes$ & 0 & $\ldots$ & 0 & $\otimes$ & $\ldots$ & $\otimes$ & $\otimes$ & $\ldots$ & 0 & $\otimes$ \\
\hline & $\vdots$ & $\vdots$ & $\vdots$ & $\vdots$ & $\vdots$ & $\vdots$ & $\vdots$ & $\vdots$ & $\vdots$ & $\vdots$ & $\vdots$ & $\ldots$ & $\vdots$ & $\vdots$ & $\vdots$ & $\vdots$ & $\because$ & $\vdots$ & $\vdots$ & $\ldots$ & $\vdots$ & $\vdots$ & $\ldots$ & $\vdots$ & $\vdots$ \\
\hline$v_{3, n}^{+}$ & $-\otimes$ & 0 & 0 & 0 & 0 & 0 & $\ldots$ & 0 & 0 & 0 & 0 & $\ldots$ & $-\otimes$ & $-\otimes$ & $-\otimes$ & 0 & $\ldots$ & 0 & $\otimes$ & $\ldots$ & $\otimes$ & 0 & $\ldots$ & $\otimes$ & $\otimes$ \\
\hline$v_{3,}$ & 0 & $-\otimes$ & 0 & 0 & 0 & 0 & $\ldots$ & 0 & 0 & 0 & 0 & $\ldots$ & $-\otimes$ & $-\otimes$ & 0 & $-\otimes$ & $\ldots$ & $-\otimes$ & 0 & $\ldots$ & 0 & $\otimes$ & $\ldots$ & $\otimes$ & $\otimes$ \\
\hline : & $\vdots$ & $\vdots$ & $\vdots$ & $\vdots$ & $\vdots$ & $\vdots$ & $\ldots$ & $\vdots$ & $\vdots$ & $\vdots$ & $\vdots$ & $\ldots$ & $\vdots$ & $\vdots$ & $\vdots$ & $\vdots$ & $\ldots$ & $\vdots$ & $\vdots$ & $\ddots$ & $\vdots$ & $\vdots$ & $\ldots$ & $\vdots$ & $\vdots$ \\
\hline$v_{k,}^{+}$ & $-\otimes$ & 0 & 0 & 0 & 0 & 0 & $\ldots$ & $-\otimes$ & 0 & $-\otimes$ & $-\otimes$ & $\ldots$ & 0 & 0 & $-\otimes$ & $-\otimes$ & $\ldots$ & $-\otimes$ & 0 & $\ldots$ & 0 & $\otimes$ & $\ldots$ & $\otimes$ & 0 \\
\hline & 0 & $-\otimes$ & 0 & 0 & 0 & 0 & $\ldots$ & 0 & $-\otimes$ & $-\otimes$ & $-\otimes$ & $\ldots$ & 0 & 0 & $-\otimes$ & $-\otimes$ & $\ldots$ & 0 & $-\otimes$ & $\ldots$ & $-\otimes$ & 0 & $\ldots$ & 0 & $\otimes$ \\
\hline & $\vdots$ & $\vdots$ & $\vdots$ & $\vdots$ & $\vdots$ & $\vdots$ & $\ldots$ & $\vdots$ & $\vdots$ & $\vdots$ & $\vdots$ & $\ldots$ & $\vdots$ & $\vdots$ & $\vdots$ & $\vdots$ & $\ldots$ & $\vdots$ & $\vdots$ & $\ldots$ & $\vdots$ & $\vdots$ & $\ddots$ & $\vdots$ & \\
\hline & $-\otimes$ & 0 & 0 & 0 & 0 & 0 & $\ldots$ & $-\otimes$ & 0 & 0 & 0 & $\ldots$ & $-\otimes$ & $-\otimes$ & $-\otimes$ & 0 & $\ldots$ & $-\otimes$ & $-\otimes$ & $\ldots$ & $-\otimes$ & 0 & $\ldots$ & 0 & $\otimes$ \\
\hline & 0 & $-\otimes$ & 0 & 0 & 0 & 0 & $\ldots$ & 0 & $-\otimes$ & 0 & 0 & $\ldots$ & $-\otimes$ & $-\otimes$ & 0 & $-\otimes$ & $\ldots$ & $-\otimes$ & $-\otimes$ & & 0 & $-\otimes$ & & $-\otimes$ & 0 \\
\hline
\end{tabular}

In the Kirchhoff contradiction function matrix above, the element of the matrix is every contradiction function

$$
\otimes_{i j}=\frac{p_{i j}(t)}{f_{i j}(t)}
$$

on the corresponding edges $E_{i j}$ (if the edge $E_{i j}$ exists). The time $t$ in vertex sequence symbols of the first row and the first column is deleted to save page space. 
3.2.4 Power Capacity Function Matrix $M_{K p c}=M_{K p} \times M_{K f}$

\begin{tabular}{|c|c|c|c|c|c|c|c|c|c|c|c|c|c|c|c|c|c|c|c|c|c|c|c|c|c|}
\hline \multicolumn{26}{|c|}{$M_{K p c}=$} \\
\hline 0 & $v_{+1}$ & $v_{-1}$ & $v_{1,1}^{+}$ & $v_{1,1}^{-}$ & $v_{2,1}^{+}$ & $v_{2,1}^{-}$ & $\cdots$ & $v_{k, 1}^{+}$ & $v_{k, 1}^{-}$ & $v_{2,2}^{+}$ & $v_{2,2}^{-}$ & $\cdots$ & $v_{2, n}^{+}$ & $v_{2, n}^{-}$ & $v_{3,2}^{+}$ & $v_{3,2}^{-}$ & $\cdots$ & $v_{3, n}^{+}$ & $v_{3, n}^{-}$ & $\cdots$ & $v_{k, 2}^{+}$ & $v_{k, 2}^{-}$ & $\cdots$ & $v_{k, n}^{+}$ & $v_{k, n}^{-}$ \\
\hline$v_{+1}$ & 0 & $2 I$ & $\otimes$ & 0 & $\otimes$ & 0 & $\cdots$ & $\otimes$ & 0 & $\otimes$ & 0 & $\cdots$ & $\otimes$ & 0 & $\otimes$ & 0 & $\cdots$ & $\otimes$ & 0 & $\cdots$ & $\otimes$ & 0 & $\cdots$ & $\otimes$ & 0 \\
\hline$v_{-1}$ & $-2 I$ & 0 & 0 & $\otimes$ & 0 & $\otimes$ & $\ldots$ & 0 & $\otimes$ & 0 & $\otimes$ & $\ldots$ & 0 & $\otimes$ & 0 & $\otimes$ & $\ldots$ & 0 & $\otimes$ & $\ldots$ & 0 & $\otimes$ & $\ldots$ & 0 & $\otimes$ \\
\hline$v_{1,1}^{+}$ & $-\otimes$ & 0 & 0 & $\otimes$ & $\otimes$ & $\otimes$ & $\ldots$ & $\otimes$ & $\otimes$ & 0 & 0 & $\ldots$ & 0 & 0 & 0 & 0 & $\ldots$ & 0 & 0 & $\ldots$ & 0 & 0 & $\ldots$ & 0 & 0 \\
\hline$v_{1,1}^{-}$ & 0 & $-\otimes$ & $-\otimes$ & 0 & $\otimes$ & $\otimes$ & $\cdots$ & $\otimes$ & $\otimes$ & 0 & 0 & $\cdots$ & 0 & 0 & 0 & 0 & $\cdots$ & 0 & 0 & $\ldots$ & 0 & 0 & $\ldots$ & 0 & 0 \\
\hline$v_{2,1}^{+}$ & $-\otimes$ & 0 & $-\otimes$ & $-\otimes$ & 0 & $\otimes$ & $\cdots$ & $\otimes$ & $\otimes$ & $\otimes$ & 0 & $\cdots$ & $\otimes$ & 0 & 0 & 0 & $\cdots$ & 0 & 0 & $\cdots$ & 0 & 0 & $\cdots$ & 0 & 0 \\
\hline$v_{2,1}^{-}$ & 0 & $-\otimes$ & $-\otimes$ & $-\otimes$ & $-\otimes$ & 0 & $\cdots$ & $\otimes$ & $\otimes$ & 0 & $\otimes$ & $\cdots$ & 0 & $\otimes$ & 0 & 0 & $\cdots$ & 0 & 0 & $\cdots$ & 0 & 0 & $\cdots$ & 0 & 0 \\
\hline$\vdots$ & $\vdots$ & $\vdots$ & $\vdots$ & $\vdots$ & $\vdots$ & $\vdots$ & $\ddots$ & $\vdots$ & $\vdots$ & $\vdots$ & $\vdots$ & $\cdots$ & $\vdots$ & $\vdots$ & $\vdots$ & $\vdots$ & $\cdots$ & $\vdots$ & $\vdots$ & $\cdots$ & $\vdots$ & $\vdots$ & $\cdots$ & $\vdots$ & $\vdots$ \\
\hline$v_{k, 1}^{+}$ & $-\otimes$ & 0 & $-\otimes$ & $-\otimes$ & $-\otimes$ & $-\otimes$ & $\ldots$ & 0 & $\otimes$ & 0 & 0 & $\ldots$ & 0 & 0 & 0 & 0 & $\ldots$ & 0 & 0 & $\ldots$ & $\otimes$ & 0 & $\ldots$ & $\otimes$ & 0 \\
\hline$v_{k, 1}^{-}$ & 0 & $-\otimes$ & $-\otimes$ & $-\otimes$ & $-\otimes$ & $-\otimes$ & $\cdots$ & $-\otimes$ & 0 & 0 & 0 & $\cdots$ & 0 & 0 & 0 & 0 & $\cdots$ & 0 & 0 & $\ldots$ & 0 & $\otimes$ & $\cdots$ & 0 & $\otimes$ \\
\hline$v_{2,2}^{+}$ & $-\otimes$ & 0 & 0 & 0 & $-\otimes$ & 0 & $\cdots$ & 0 & 0 & 0 & $\otimes$ & $\cdots$ & $\otimes$ & 0 & $\otimes$ & $\otimes$ & $\cdots$ & 0 & 0 & $\ldots$ & $\otimes$ & $\otimes$ & $\cdots$ & 0 & 0 \\
\hline$v_{2,2}^{-}$ & 0 & $-\otimes$ & 0 & 0 & 0 & $-\otimes$ & $\ldots$ & 0 & 0 & $-\otimes$ & 0 & $\cdots$ & 0 & $\otimes$ & $\otimes$ & $\otimes$ & $\ldots$ & 0 & 0 & $\ldots$ & $\otimes$ & $\otimes$ & $\ldots$ & 0 & 0 \\
\hline$\vdots$ & $\vdots$ & $\vdots$ & $\vdots$ & $\vdots$ & $\vdots$ & $\vdots$ & $\vdots$ & $\vdots$ & $\vdots$ & $\vdots$ & $\vdots$ & $\ddots$ & $\vdots$ & $\vdots$ & $\vdots$ & $\vdots$ & $\ldots$ & $\vdots$ & $\vdots$ & $\ldots$ & $\vdots$ & $\vdots$ & $\ldots$ & $\vdots$ & $\vdots$ \\
\hline$v_{2, n}^{+}$ & $-\otimes$ & 0 & 0 & 0 & $-\otimes$ & 0 & $\cdots$ & 0 & 0 & $-\otimes$ & 0 & $\cdots$ & 0 & $\otimes$ & 0 & 0 & $\cdots$ & $\otimes$ & $\otimes$ & $\ldots$ & 0 & 0 & $\cdots$ & $\otimes$ & $\otimes$ \\
\hline$v_{2, n}^{-}$ & 0 & $-\otimes$ & 0 & 0 & 0 & $-\otimes$ & $\cdots$ & 0 & 0 & 0 & $-\otimes$ & $\cdots$ & $-\otimes$ & 0 & 0 & 0 & $\cdots$ & $\otimes$ & $\otimes$ & $\cdots$ & 0 & 0 & $\cdots$ & $\otimes$ & $\otimes$ \\
\hline$v_{3,2}^{+}$ & $-\otimes$ & 0 & 0 & 0 & 0 & 0 & $\cdots$ & 0 & 0 & $-\otimes$ & $-\otimes$ & $\cdots$ & 0 & 0 & 0 & $\otimes$ & $\cdots$ & $\otimes$ & 0 & $\ldots$ & $\otimes$ & $\otimes$ & $\cdots$ & $\otimes$ & 0 \\
\hline$v_{3,2}^{-}$ & 0 & $-\otimes$ & 0 & 0 & 0 & 0 & $\ldots$ & 0 & 0 & $-\otimes$ & $-\otimes$ & $\ldots$ & 0 & 0 & $-\otimes$ & 0 & $\ldots$ & 0 & $\otimes$ & $\ldots$ & $\otimes$ & $\otimes$ & $\ldots$ & 0 & $\otimes$ \\
\hline$\vdots$ & $\vdots$ & $\vdots$ & $\vdots$ & $\vdots$ & $\vdots$ & $\vdots$ & $\vdots$ & $\vdots$ & $\vdots$ & $\vdots$ & $\vdots$ & $\ldots$ & $\vdots$ & $\vdots$ & $\vdots$ & $\vdots$ & $\ddots$ & $\vdots$ & $\vdots$ & $\ldots$ & $\vdots$ & $\vdots$ & $\ldots$ & $\vdots$ & $\vdots$ \\
\hline$v_{3, n}^{+}$ & $-\otimes$ & 0 & 0 & 0 & 0 & 0 & $\ldots$ & 0 & 0 & 0 & 0 & $\ldots$ & $-\otimes$ & $-\otimes$ & 0 & 0 & $\ldots$ & 0 & $\otimes$ & $\ldots$ & $\otimes$ & 0 & $\ldots$ & $\otimes$ & $\otimes$ \\
\hline$v_{3, n}^{-}$ & 0 & $-\otimes$ & 0 & 0 & 0 & 0 & $\cdots$ & 0 & 0 & 0 & 0 & $\cdots$ & $-\otimes$ & $-\otimes$ & 0 & $-\otimes$ & $\ldots$ & $-\otimes$ & 0 & $\cdots$ & 0 & $\otimes$ & $\ldots$ & $\otimes$ & $\otimes$ \\
\hline$\vdots$ & $\vdots$ & $\vdots$ & $\vdots$ & $\vdots$ & $\vdots$ & $\vdots$ & $\ldots$ & $\vdots$ & $\vdots$ & $\vdots$ & $\vdots$ & $\ldots$ & $\vdots$ & $\vdots$ & $\vdots$ & $\vdots$ & $\ldots$ & $\vdots$ & $\vdots$ & $\ddots$ & $\vdots$ & $\vdots$ & $\ldots$ & $\vdots$ & $\vdots$ \\
\hline$v_{k, 2}^{+}$ & $-\otimes$ & 0 & 0 & 0 & 0 & 0 & $\ldots$ & $-\otimes$ & 0 & $-\otimes$ & $-\otimes$ & $\ldots$ & 0 & 0 & $-\otimes$ & $-\otimes$ & $\ldots$ & $-\otimes$ & 0 & $\ldots$ & 0 & $\otimes$ & $\ldots$ & $\otimes$ & 0 \\
\hline$v_{k, 2}^{-}$ & 0 & $-\otimes$ & 0 & 0 & 0 & 0 & $\ldots$ & 0 & $-\otimes$ & $-\otimes$ & $-\otimes$ & $\ldots$ & 0 & 0 & $-\otimes$ & $-\otimes$ & $\ldots$ & 0 & $-\otimes$ & $\ldots$ & $-\otimes$ & 0 & $\ldots$ & 0 & $\otimes$ \\
\hline$\vdots$ & $\vdots$ & $\vdots$ & $\vdots$ & $\vdots$ & $\vdots$ & $\vdots$ & $\cdots$ & $\vdots$ & $\vdots$ & $\vdots$ & $\vdots$ & $\cdots$ & $\vdots$ & $\vdots$ & $\vdots$ & $\vdots$ & $\cdots$ & $\vdots$ & $\vdots$ & $\cdots$ & $\vdots$ & $\vdots$ & $\ddots$ & $\vdots$ & $\vdots$ \\
\hline$v_{k, n}^{+}$ & $-\otimes$ & 0 & 0 & 0 & 0 & 0 & $\cdots$ & $-\otimes$ & 0 & 0 & 0 & $\cdots$ & $-\otimes$ & $-\otimes$ & $-\otimes$ & 0 & $\ldots$ & $-\otimes$ & $-\otimes$ & $\ldots$ & $-\otimes$ & 0 & $\cdots$ & 0 & $\otimes$ \\
\hline$v_{k, n}^{-}$ & 0 & $-\otimes$ & 0 & 0 & 0 & 0 & $\ldots$ & 0 & $-\otimes$ & 0 & 0 & $\ldots$ & $-\otimes$ & $-\otimes$ & 0 & $-\otimes$ & $\ldots$ & $-\otimes$ & $-\otimes$ & $\ldots$ & 0 & $-\otimes$ & $\ldots$ & $-\otimes$ & 0 \\
\hline
\end{tabular}

In the Kirchhoff power capacity matrix above, the element of the matrix is every power capacity function

$$
\otimes_{i j}=p_{i j}(t) f_{i j}(t)
$$

on corresponding edge $E_{i j}$ (if the edge $E_{i j}$ exists). The time $t$ in vertex sequence symbols of the first row and the first column is deleted to save page space.

3.2.5 Work And Energy Definite Integral Matrix $M_{K w e}$

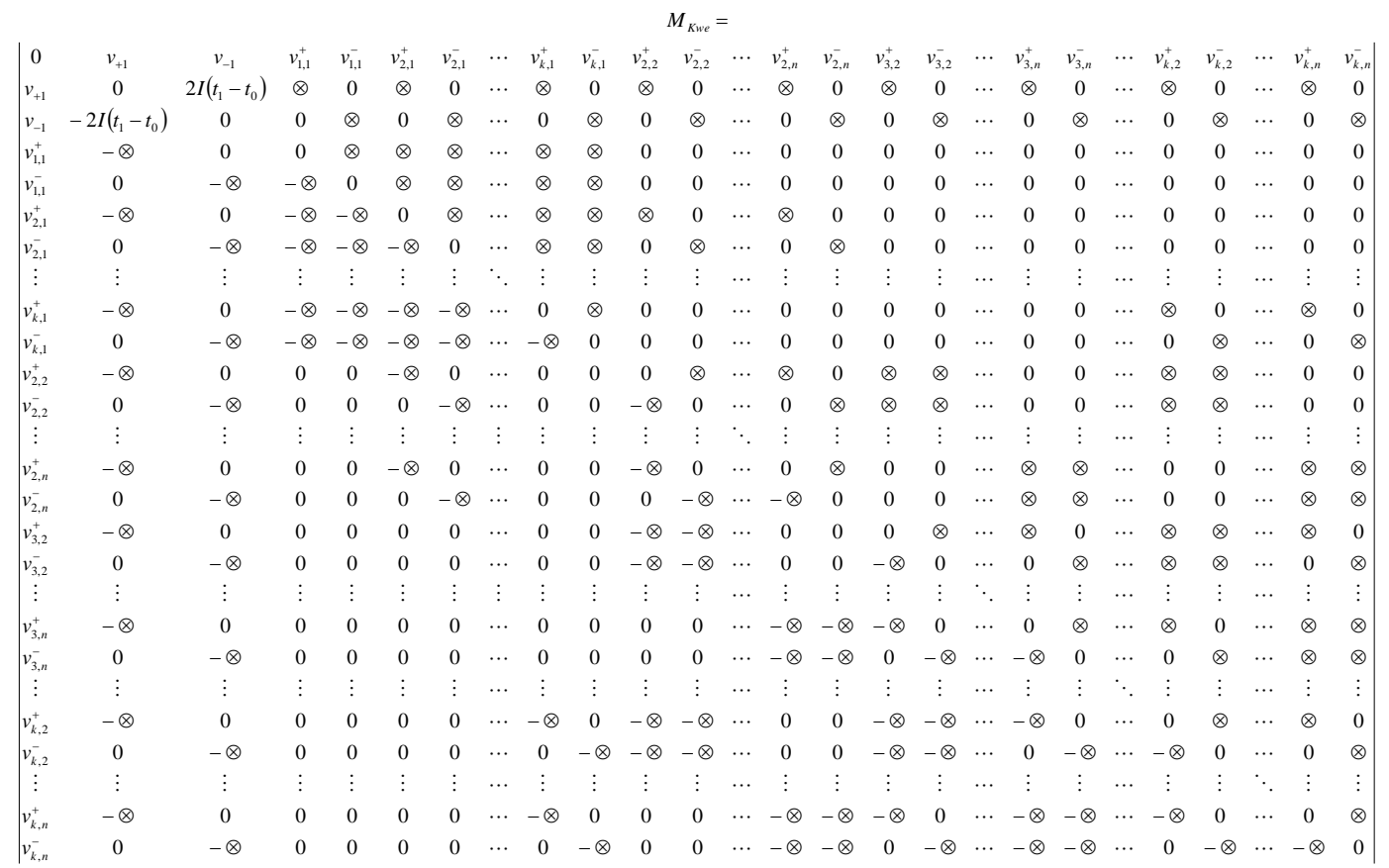

In the Kirchhoff work and energy definite integral matrix above, the element of the matrix is 


$$
\bigotimes_{i j}=\int_{t_{0}}^{t_{1}} p_{i j}(t) f_{i j}(t) d t
$$

on the corresponding edges $E_{i j}$ (if the edge $E_{i j}$ exists). The time $t$ in vertex sequence symbols of the first row and the first column is deleted to save page space.

The definite integral matrix below

$$
M_{K w e}=\int_{t_{0}}^{t_{1}} M_{K p c} d t=\int_{t_{0}}^{t_{1}}\left(M_{K p} \times M_{K f}\right) d t=\left|\int_{t_{0}}^{t_{1}} p_{i j}(t) f_{i j}(t) d t\right|
$$

is the matrix expression for Kirchhoff work and energy definite integral.

\subsubsection{The Basic Properties of Kirchhoff Matrices}

A. These Kirchhoff matrices above are all "every element on main diagonal is zero";

B. These Kirchhoff matrices above are all axis-symmetrical on the main diagonal taken as symmetrical axis, therefor the Kirchhoff matrices above are all triangular matrix;

C. When a graph is named a inverse graph $G^{-1}$ about graph $G$, if and only if the $G^{-1}$ is isomorphic-equality to the graph $G$, but its direction of every edge is inverse. The Kirchhoff matrix above has been divided into two graphs by the main diagonal, in which one is the Kirchhoff graph and another is the inverse graph of the Kirchhoff graph. With another word, as a boundary by the main diagonal, the matrix can be divided into two parts: the right-upper and the left-lower. The corresponding graph of right-upper and left-lower are inverse graph each other;

D. The blocking of Kirchhoff matrices

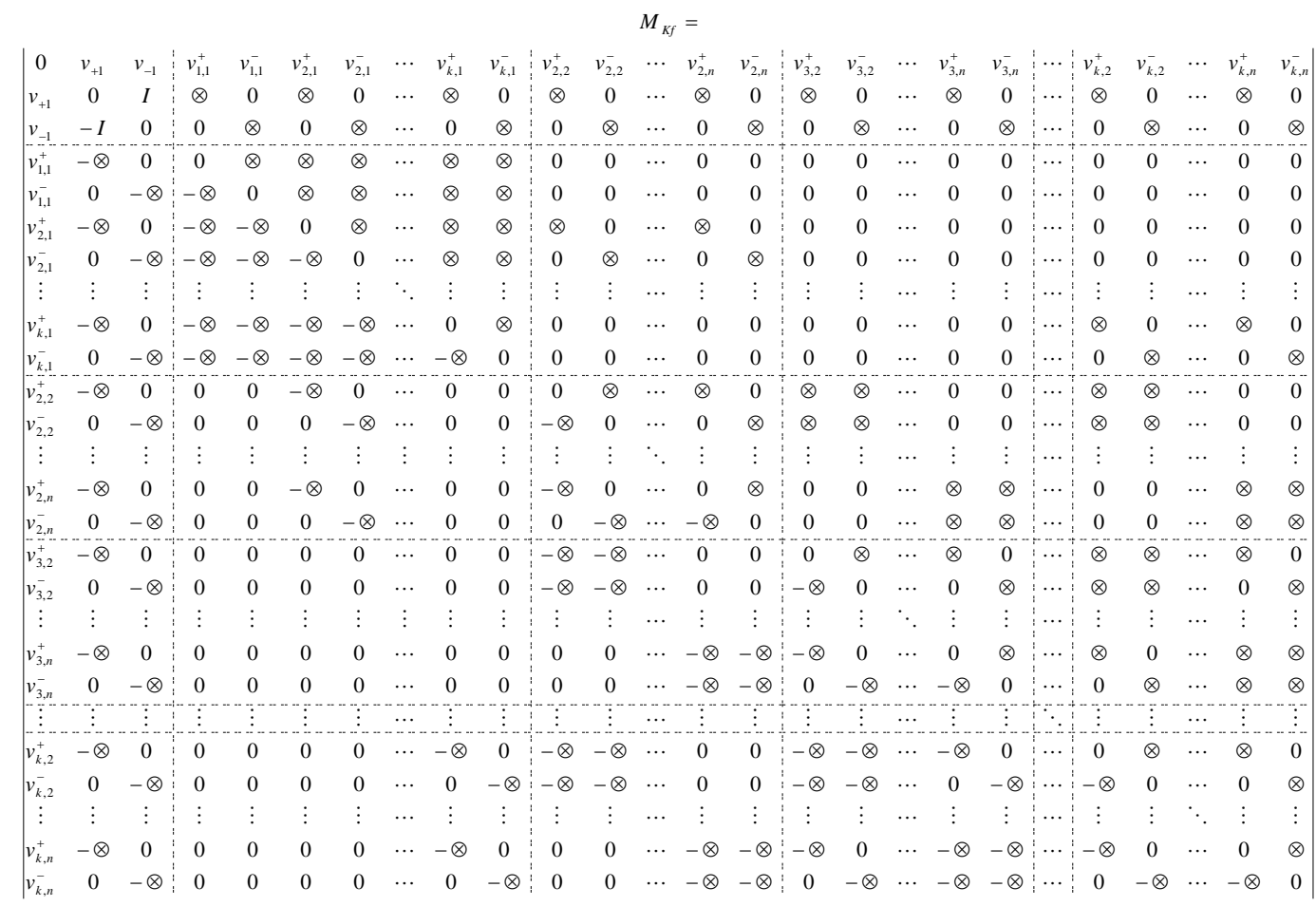

Therefor the flow function Kirchhoff blocked matrix is as below: 


$$
M_{K f}=\left\|\begin{array}{cccccc}
A_{3 \times 3} & B_{3 \times 2 k} & C_{2,3 \times 2(n-1)} & C_{3,3 \times 2(n-1)} & \cdots & C_{k, 3 \times 2(n-1)} \\
B_{2 k \times 3}^{T} & B_{4 k^{2}} & D_{2,2 k \times 2(n-1)} & D_{3,2 k \times 2(n-1)} & \cdots & D_{k, 2 k \times 2(n-1)} \\
C_{2,2(n-1) \times 3}^{T} & D_{2,2(n-1) \times 2 k}^{T} & C_{2,4(n-1)^{2}} & C_{2 \oplus 3,4(n-1)^{2}} & \cdots & C_{2 \oplus k, 4(n-1)^{2}} \\
C_{3,2(n-1) \times 3}^{T} & D_{3,2(n-1) \times 2 k}^{T} & C_{3 \oplus 2,4(n-1)^{2}}^{T} & C_{3,4(n-1)^{2}} & \cdots & C_{3 \oplus k, 4(n-1)^{2}} \\
\vdots & \vdots & \vdots & \vdots & \ddots & \vdots \\
C_{k, 2(n-1) \times 3}^{T} & D_{k, 2(n-1) \times 2 k}^{T} & C_{k \oplus 2,4(n-1)^{2}}^{T} & C_{k \oplus 3,4(n-1)^{2}}^{T} & \cdots & C_{k, 4(n-1)^{2}}
\end{array}\right\|
$$

The matrix blocks on the main diagonal, such as $A_{3 \times 3}, B_{4 k^{2}}$, is the $k$-dimensional primary sub-graph, and the other matrix blocks on the main diagonal, such as $C_{2,4(n-1)^{2}}, \cdots, C_{p, 4(n-1)^{2}}$ are the secondary sub-graph shown in the paper(Yaozhi Jiang., 2018). The blocked matrix's sub-matrix those are axis-symmetrical on main diagonal is transposed matrix each other.

We have blocked only on the Kirchhoff flow function matrix, actually we can block on all Kirchhoff matrices defined above, if the blocking is necessary to our calculation;

E. The calculating for every vertex flow function in Kirchhoff matrix

First, we define a Square-ruler when we move from the first place element of flow function matrix one by one follow the row (or column), if we meet the element zero in main diagonal then we turn from the element zero to follow the column (or row), this looks like a light reflected by the main diagonal, except the $v_{+1}$ row or column and $v_{k, n}^{-1}$ row or column.

We have known that the every row element or every column element in the Kirchhoff flow function matrix is the adjacent relationship from first place vertex of row or column to the other vertexes on the same row or column. Therefor we can obtain the below:

In the Kirchhoff flow function matrix, on the the $v_{+1}$ row or column and $v_{k, n}^{-1}$ row or column, algebraic sum of the flow function on every row or every column is zero.

Or in the Kirchhoff matrix, on the other row or column, the algebraic sum of flow function on every Square-ruler is zero.

This is the matrix expression of Kirchhoff flow function law above;

F.The calculating for cycle power function in Kirchhoff matrix

At first, we show an example of Kirchhoff power function matrix and its corresponding Kirchhoff graph below. 


$$
M_{K p}=\left|\begin{array}{ccccccccccc}
0 & +1 & -1 & p_{1,1}^{+} & p_{1,2}^{+} & \cdots & p_{1, n}^{+} & p_{1,1}^{-} & p_{1,2}^{-} & \cdots & p_{1, n}^{-} \\
+1 & 0 & 2 & \otimes & \otimes & \cdots & \otimes & 0 & 0 & \cdots & 0 \\
-1 & -2 & 0 & 0 & 0 & \cdots & 0 & \otimes & \otimes & \cdots & \otimes \\
p_{1,1}^{+} & -\otimes & 0 & 0 & \otimes & \cdots & \otimes & \otimes & 0 & \cdots & 0 \\
p_{1,2}^{+} & -\otimes & 0 & -\otimes & 0 & \cdots & \otimes & 0 & \otimes & \cdots & 0 \\
\vdots & \vdots & \vdots & \vdots & \vdots & \ddots & \vdots & \vdots & \vdots & \cdots & \vdots \\
p_{1, n}^{+} & -\otimes & 0 & -\otimes & -\otimes & \cdots & 0 & 0 & 0 & \cdots & \otimes \\
p_{1,1}^{-} & 0 & -\otimes & -\otimes & 0 & \cdots & 0 & 0 & \otimes & \cdots & \otimes \\
p_{1,2}^{-} & 0 & -\otimes & 0 & -\otimes & \cdots & 0 & -\otimes & 0 & \cdots & \otimes \\
\vdots & \vdots & \vdots & \vdots & \vdots & \cdots & \vdots & \vdots & \vdots & \ddots & \vdots \\
p_{1, n}^{-} & 0 & -\otimes & 0 & 0 & \cdots & -\otimes & -\otimes & -\otimes & \cdots & 0
\end{array}\right|
$$

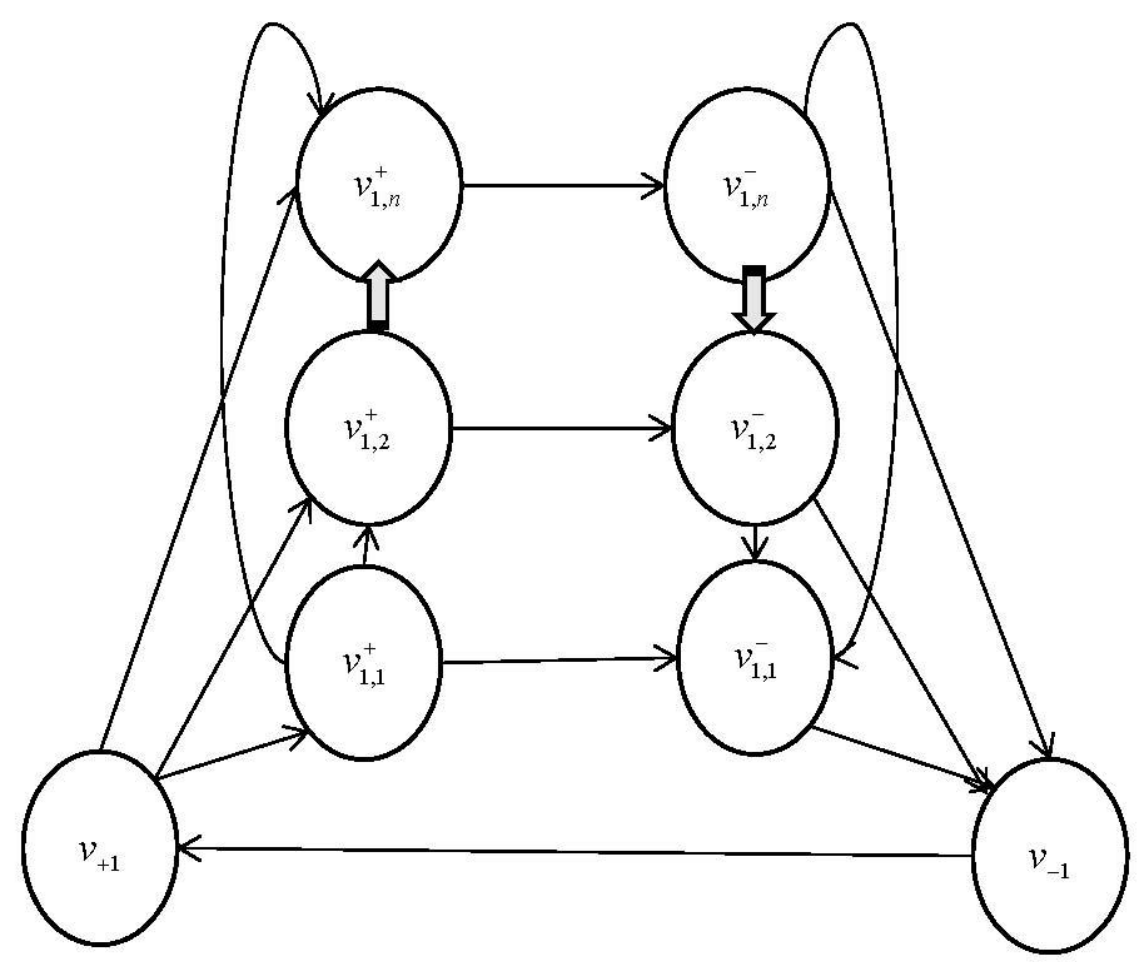

Fig.1 the example for correspondence Kirchhoff graph 
We have known that a cycle example in the Kirchhoff power function matrix is the sequence such as:

$$
v_{+1} \Rightarrow-\otimes \Rightarrow v_{1,1}^{+} \Rightarrow \otimes \Rightarrow v_{1, n}^{+} \Rightarrow-\otimes \Rightarrow v_{1, n}^{-} \Rightarrow-\otimes \Rightarrow v_{-1} \Rightarrow+2 \Rightarrow v_{+1}
$$

or

$$
v_{1,2}^{+} \Rightarrow \otimes \Rightarrow v_{1, n}^{+} \Rightarrow-\otimes \Rightarrow v_{1, n}^{-} \Rightarrow-\otimes \Rightarrow v_{1,1}^{-} \Rightarrow \otimes \Rightarrow v_{1,1}^{+} \Rightarrow-\otimes \Rightarrow v_{+1} \Rightarrow-\otimes \Rightarrow v_{1,2}^{+}
$$

The cycle defined above can be called as Kirchhoff cycle in Kirchhoff power function matrix. Therefor we have the matrix expression of Kirchhoff power function below:

The algebraic sum of all edge power function in every Kirchhoff cycle is zero.

A property might be mentioned that in the Kirchhoff cycle there are not repeating edges in the same cycle;

F. The minimal power capacity path from vertex $V_{+1}$ to vertex $V_{-1}$

Now we have another problem: how to calculate the minimal power capacity path from vertex $V_{+1}$ to vertex $V_{-1}$ ? Undoubtedly such a path exists. We can use the famous Dijkstra's Algorithm in graph theory to the power capacity matrix to solve the problem.(Berge., 1973)

Redundancy analysis for elements in Kirchhoff matrices

Redundancy analysis is for the elements in Kirchhoff contradiction function matrix. To every nonzero element in Kirchhoff contradiction function matrix, if the contradiction function value of some element is larger, it is more important. With another word, if the contradiction function value of some element is larger, its redundancy is smaller. According to this property we can delete the elements whose redundancy is larger to simplify the calculating of Kirchhoff matrices. Of cause the deleting would increase the error of true value function.

\section{Conclusion}

As shown above, author has solved the two problems: multidimensional discrete sampling system on multidimensional logic variable true value function and further properties of Kirchhoff matrices, even if they are theoretical but can be played two very important parts in the dialectical logic K-model. And this paper indicated that the dialectical logic $\mathrm{K}$-model is rather complicated.

\section{References}

Berge, C. (1973). Graph and hyper graphs[M]_Amsterdam: North-Holland Publishing Company.

Maden, A. D., Cevik, A. S., Cangul, I. N., et al. (2013). Retracted Article: On the Kirchhoff matrix, a new Kirchhoff index and the Kirchhoff energy, J Inequal Appl (2013:337). Springer International Publishing. https://doi.org/10.1186/1029-242x-2013-337

Ronald Bracewell, N. (1999). The Fourier Transform and Its Applications, (1999, third edition), Mcgraw-Hill Science/Engineering/Math.

Yaozhi Jiang (2017). Dialectical Logic K-Model: A Mathematical Model for Machine. Journal of Mathematics Research, 9(6). http://doi.org/10.5539/jmr.v9n6p82

Yaozhi Jiang (2018). Dialectical Logic K-Model: Discrete Time Dynamical Sample System, Multidimensional logic variable and Associated Database, Journal of Mathematics Research, 10(2) (accepted and to appear, first online )

\section{Copyrights}

Copyright for this article is retained by the author(s), with first publication rights granted to the journal.

This is an open-access article distributed under the terms and conditions of the Creative Commons Attribution license (http://creativecommons.org/licenses/by/4.0/). 